Document downloaded from:

http://hdl.handle.net/10251/123254

This paper must be cited as:

Miralles-Navarro, E.; Multerer, T.; Ganis, A.; Schoenlinner, B.; Prechtel, U.; Meusling, A.; Mietzner, J.... (2018). Multifunctional and compact 3D FMCW MIMO radar system with rectangular array for medium-range applications. IEEE Aerospace and Electronic Systems Magazine. 33(4):46-54. https://doi.org/10.1109/MAES.2018.160277

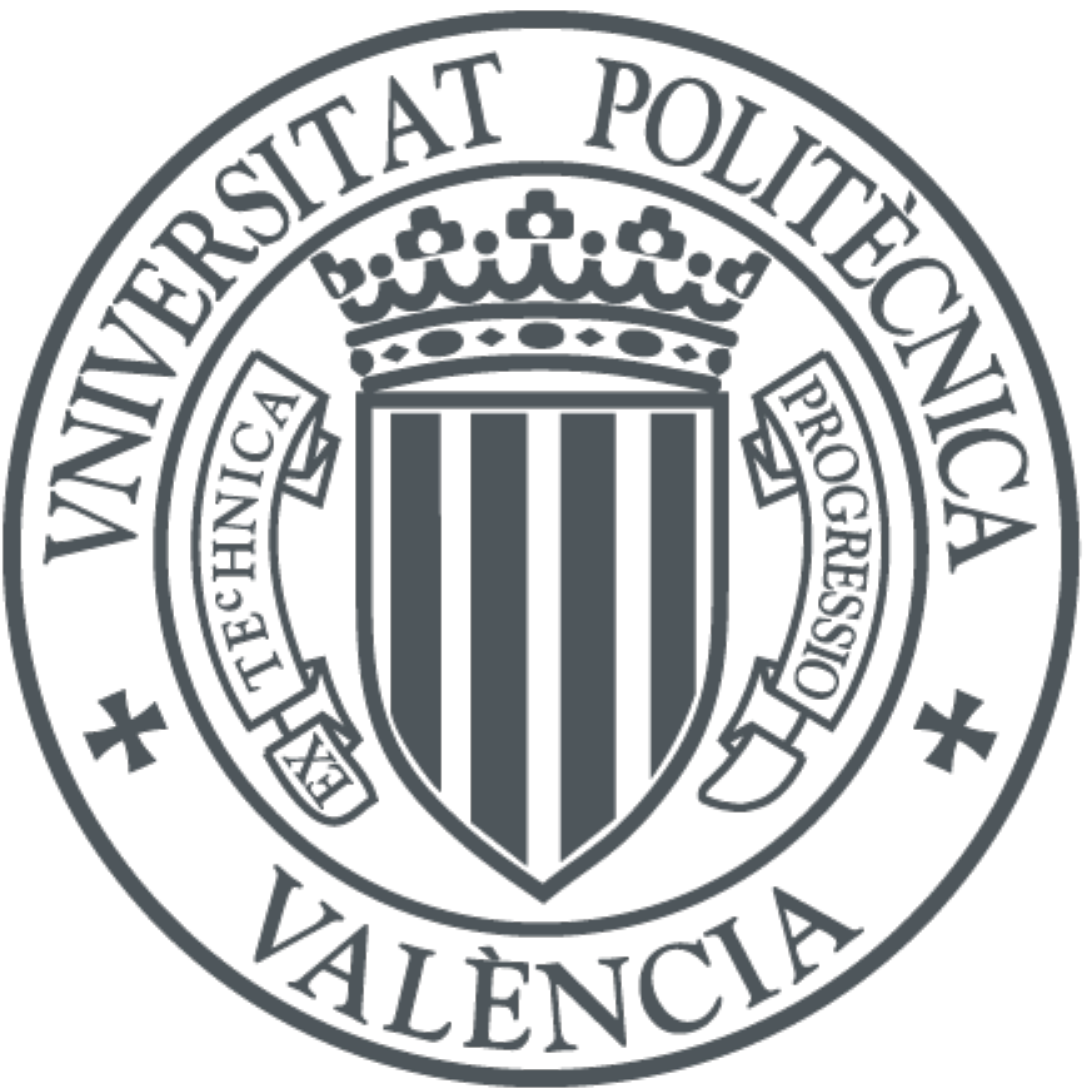

The final publication is available at

http://doi.org/10.1109/MAES.2018.160277

Copyright Institute of Electrical and Electronics Engineers

Additional Information 


\title{
Multifunctional and Compact 3D FMCW MIMO Radar System with Rectangular Array for Medium Range Applications
}

\author{
Enric Miralles*§, Thomas Multerer* ${ }^{*}$, Alexander Ganis*\|, Bernhard Schoenlinner*, \\ Ulrich Prechtel* $^{*}$, Askold Meusling ${ }^{\dagger}$, Jan Mietzner ${ }^{\ddagger}$, Christian Weckerle ${ }^{\ddagger}$, \\ Hector Esteban ${ }^{\S}$, Martin Vossiek ${ }^{\Uparrow}$, Mirko Loghill and Volker Ziegler* \\ Email: enric.miralles-navarro@airbus.com, thomas.t.multerer@airbus.com \\ * Airbus Group Innovations, Willy-Messerschmitt-Straße 1, 82024 Taufkirchen, Germany \\ $\dagger$ Airbus Defence and Space, Willy-Messerschmitt-Straße 1, 82024 Taufkirchen, Germany \\ $\ddagger$ Airbus Defence and Space Electronics and Border Security GmbH, Woerthstrasse 85, 89077 Ulm, Germany \\ $\S$ Universidad Politecnica de Valencia, Camino de Vera, s/n, 46022 Valencia, Spain \\ 『 Friedrich-Alexander Universität Erlangen-Nürnberg, Cauerstraße 9, 91058 Erlangen, Germany \\ || University of Udine, Via delle Scienze 206, 33100 Udine, Italy
}

\begin{abstract}
This paper presents an approach to combine a 3D Frequency Modulated Continuous Wave (FMCW) Multiple Input Multiple Output (MIMO) radar system with additional sensors and/or actuators, introducing multiple functions, which are integrated in a single system. A rectangular MIMO array with two rows of transmit (TX) antennas and two rows of receive (RX) antennas which are placed opposite to each other offers an empty space in the center. This allows to integrate additional devices in the unused space, like a camera or a communication system. In order to exploit synergies, a central processing unit which is capable of controlling the system and evaluating the data is installed. Furthermore, a metallic 3D printed housing, which accomplishes also the task of cooling the electronics, has been built and assembled. The antenna board consists of 16 TX and $16 \mathrm{RX}$ antennas which are placed on a single planar Printed Circuit Board (PCB). Orthogonality of the TX signals is obtained through Time-Division Multiplexing (TDM). The radar sensor working frequency range spans between $16 \mathrm{GHz}$ and 17 GHz. The system operates with 3D Fast Fourier Transform (FFT) beam forming at the RX side. To show the advantages of a second functionality, a MIMO radar system combined with a camera and a gimbal for target tracking are exposed. For this multifunctional system, detailed descriptions of the hardware and software architecture, measurements and 3D radar images are presented in this paper.
\end{abstract}

Index Terms-Multiple Input Multiple Output (MIMO), radar, Frequency Modulated Continuous Wave (FMCW), Time-Division Multiplexing (TDM), three-dimensional, 3D, FFT, beamforming, Digital Beamforming, Printed Circuit Board (PCB), Actuator, 3D Printing, Multi-functionality, Camera, Gimbal.

\section{INTRODUCTION}

Ever since the era of autonomous systems started, multisensor platforms have become a key topic in aerospace, automotive and robotics industries. Multifunctional systems integrate more than one sensor and/or actuator in one device and are capable of creating synergies between them. As

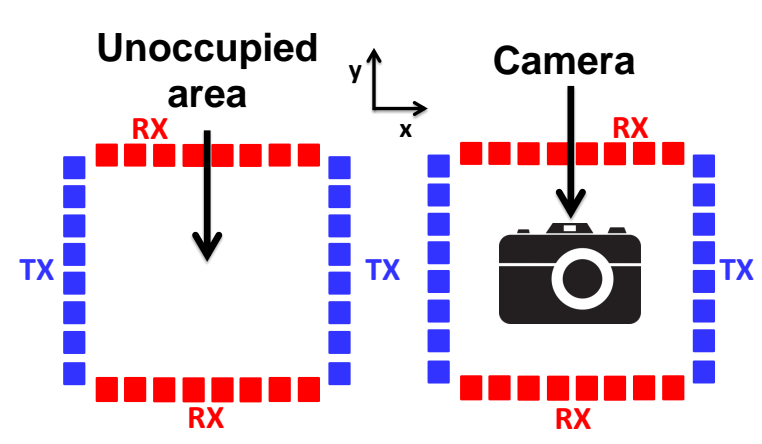

Fig. 1. Visualization of a rectangular MIMO array with unoccupied space in the center (left) and a camera (right).

a consequence, their performance is superior compared to single-sensor systems, for different reasons [1] [2] [3].

First of all, these systems can capture, process and merge information from different perspectives that can complement each other. Second, a more compact approach is possible, because the hardware required for additional functionalities can be integrated in unoccupied spaces of the main system. Third, a reduction of the costs is possible in a multifunctional architecture, since part of the hardware can be shared. For instance, the signal processing unit (Advanced RISC Machines (ARM) and Field Programmable Gate Array (FPGA)) or the power supply unit. Sharing hardware components can also lead to a reduction in both power consumption and weight. To summarize, Size, Weight and Power (SWaP) optimization can be achieved with a proper design of a multifunctional system. This is of vital importance and highly profitable for many applications.

One of the most popular sensors technologies is radar 


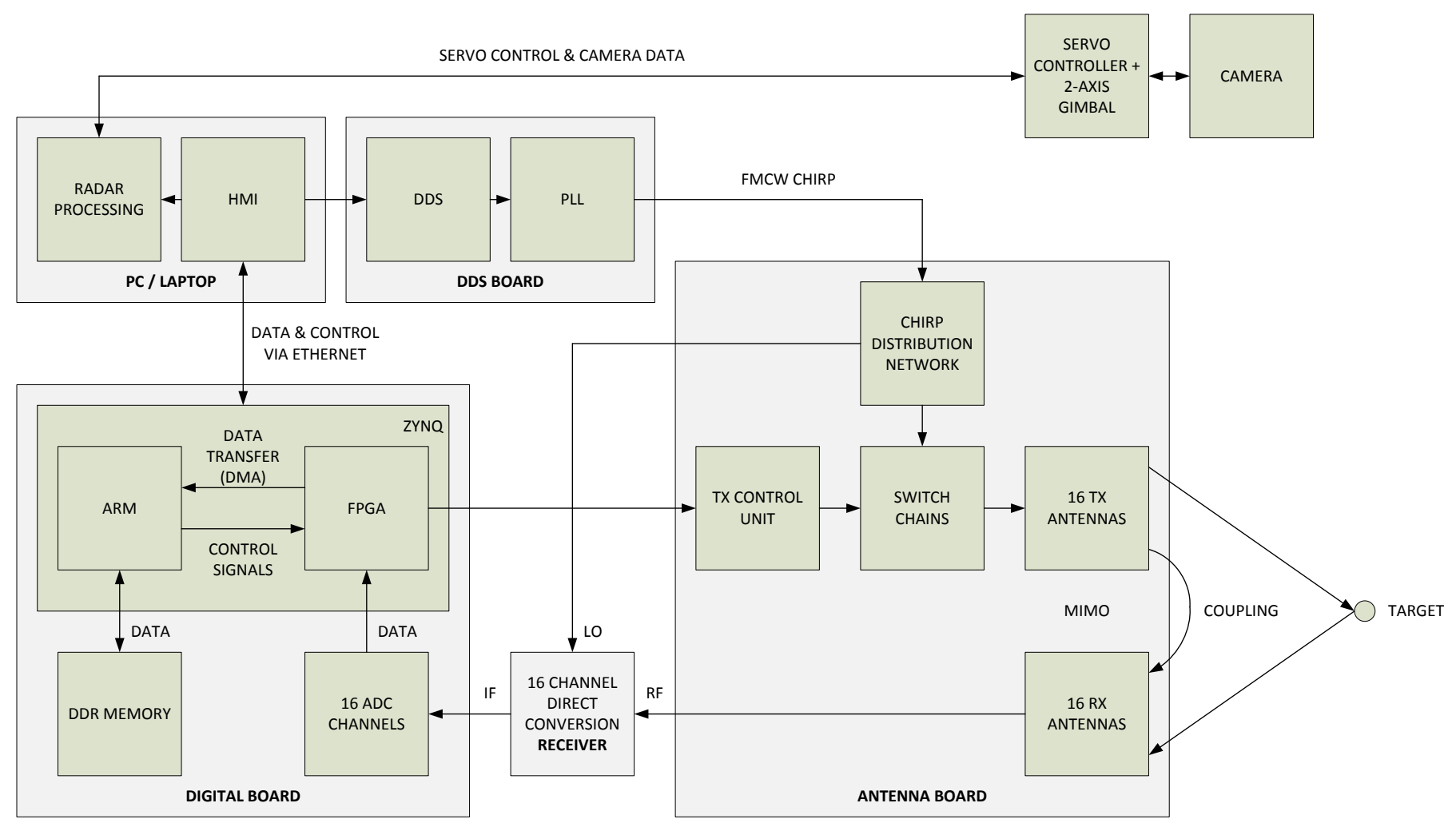

Fig. 2. Block diagram of the 3D imaging FMCW 16x16 MIMO radar demonstrator. The main components are the antenna board, the digital board, the DDS board, the receiver, the laptop and the camera.

[4], due to its all-weather capabilities, its ability to penetrate material and to provide a 3D sensing of range, azimuth and elevation. A radar sensor can be combined with additional functionalities, depending on the task, for example: a light source (torch or laser pointer), a weapon, antennas for communication and a (thermo-) camera. This paper presents a system which combines a MIMO radar [5], and a camera mounted on a gimbal. This combination of sensors can ease the task of change detection and classification of targets [6] [7].

Some work has already been done in this field. For example, a 2D FMCW [8] MIMO radar combined with a camera is presented in [9]. In this architecture, the camera and the radar are not integrated into one single system. It is rather multiple systems operating together than one multifunctional system. Furthermore, the used radar sensor is not able to generate elevation information. In addition, the presented device is bulky and not portable, which is highly required for the targeted application. Another approach is shown in [10]. Here, the system is intended for indoor use and short range applications. Like the previous system, it cannot estimate the elevation of the target. Nevertheless, an increase in tracking performance is shown by the combination of radar and camera capabilities through data fusion. Another system which deals with the advantages of having a radar and a camera for object classification is presented in [6]. This system, as well as the others, does not create a full 3D reconstruction of the observed scene. Moreover, the chosen camera has a low resolution, which can be appropriate in an automotive context, but not for medium range applications. The advantages of having a multifunctional system in a naval environment are well described in [11].

In this paper, a 3D FMCW MIMO radar in combination with a camera and a two axis gimbal, placed in the center of the MIMO array, is presented. For a determined number of TX and RX modules, a MIMO radar offers a higher angular resolution compared to a conventional phased array radar [12]. Consequently, it is smaller, lighter and costs less. The MIMO architecture uses the spatial separation of the antennas to create a so called virtual array which can be calculated via a discrete convolution [13]. The size of the resulting virtual array, and not the physical array, determines the angular resolution of the MIMO radar system. If the TX and RX antennas of the array are placed in a rectangular configuration, the center of the antenna aperture is unoccupied. This area is used to place the camera and the gimbal (see Fig. 1). With the gimbal it is possible to steer the camera to the target detected by the radar. The antenna board consists of $16 \mathrm{TX}$ and $16 \mathrm{RX}$ antennas. The radar operates in the frequency band from $16 \mathrm{GHz}$ to $17 \mathrm{GHz}$ (Ku-band), with an operational bandwidth of $1 \mathrm{GHz}$. The TX signal orthogonality is achieved by employing TDM. A 3D printed housing which protects the circuitry and acts as a cooling system for the electronic, has been fabricated and assembled.

The target application of the multifunctional 3D FMCW 


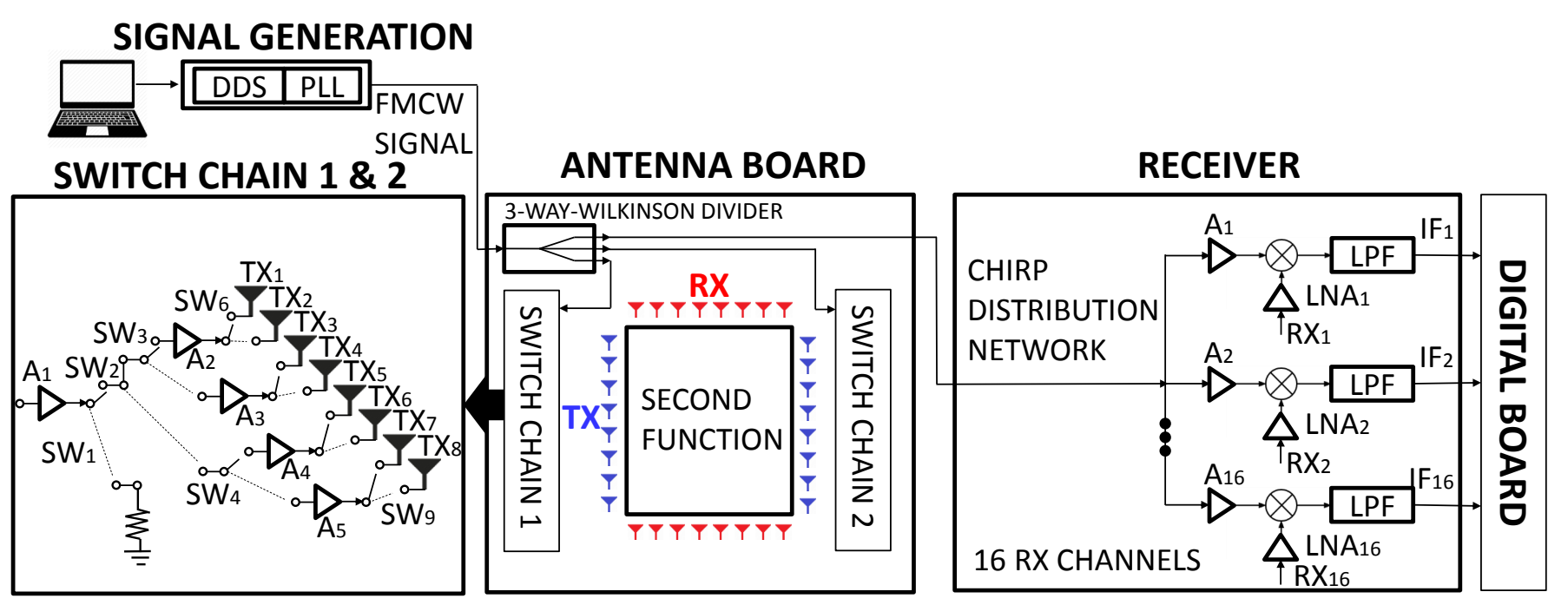

Fig. 3. Component level block diagrams of the antenna board and the receiver with schematic of the switch chains.

MIMO radar is ground-based surveillance of stationary widezones and infrastructures such as high-security or hazardous areas. These could be chemical or nuclear power plants, energy transmission infrastructure, fuel pipelines and several other applications, in which a compact and easily deployable platform is highly desired.

\section{SYSTEM ARCHITECTURE}

The top-level system architecture of the MIMO radar demonstrator is presented in the form of a block diagram in Fig. 2. The main components are the antenna board, the digital board, the Direct Digital Synthesis (DDS) board, the receiver, the laptop and the camera. The modular approach of the 3D printed housing, easily allows to add and change hardware components. The following part describes the block diagram and explains how the system works.

From the laptop, the DDS board and hence the Phase Locked Loop (PLL) can be configured to create the desired FMCW chirp. The most important parameters are the chirp length in time and the bandwidth. The chirp is then transmitted to the antenna board, where it is distributed to two switch chains and the receiver board using a 3-way Wilkinson divider, as it can be seen in Fig. 3.

First, each switch chain can select between eight TX antennas or a matched load, enabling a TDM configuration and thus orthogonality of the signals. This means that all TX antennas are switched consecutively within one MIMO cycle. Second, the receiver uses the originally sent chirp as Local Oscillator (LO) signal to down-convert the 16 received delayed chirps. This way, the beat frequencies (associated with the range information) of the target can be extracted.

A proprietary ZYNQ(Xilinx, Inc.) based platform, which includes a FPGA and an ARM processor, serves as the interface between the Analog to Digital Converters (ADCs) and the laptop performing the acquisition of digital data. The resulting signals are digitized by 16 ADCs, which are interfaced to the FPGA. The data is first stored in the FPGA and then transferred to the Double Data Rate (DDR) memory of the system via Direct Memory Access (DMA). From the digital board, the data is transferred to the laptop via Ethernet. The whole process of the data capturing is triggered by the Human Machine Interface (HMI). Once started, the TX control unit is commanded by the ZYNQ which runs a program on the ARM processor. The TX control unit sets the proper bit combination for the switch matrices in order to select the TX antenna.

The radar processing is carried out on the laptop, where digital beam-forming techniques are applied in order to extract the target position in range, azimuth and elevation. The target position is sent to the servo controller, which creates the steering signals for a two axis gimbal, where the (zoom-) camera is mounted. The camera can then take a close-up picture or video of the target and send it back to the HMI. The image can then be possibily used to classify the target or to do further image processing.

\section{RADAR HARDWARE}

This section introduces the main blocks of the radar hardware including the antenna board, the receiver board and the signal generation. The circuit level block diagrams are shown in Fig. 3.

The antenna board includes in a single planar PCB, 32 radiating elements, 16 for transmit TX (in blue) and 16 for receive RX (in red). They are placed in a rectangular configuration, in such a manner, that an unoccupied surface at the center of the array arises. In order to achieve the desired operational bandwidth and the desired range resolution, Resonant Slot Patch Antenna (RSPA) instead of traditional patch antennas are incorporated [14]. A three way Wilkinson divider equally distributes the FMCW ramp, that is generated by the DDS and PLL, to the two independent switch chains and the receiver. The two switch chains are able to select the proper antenna 


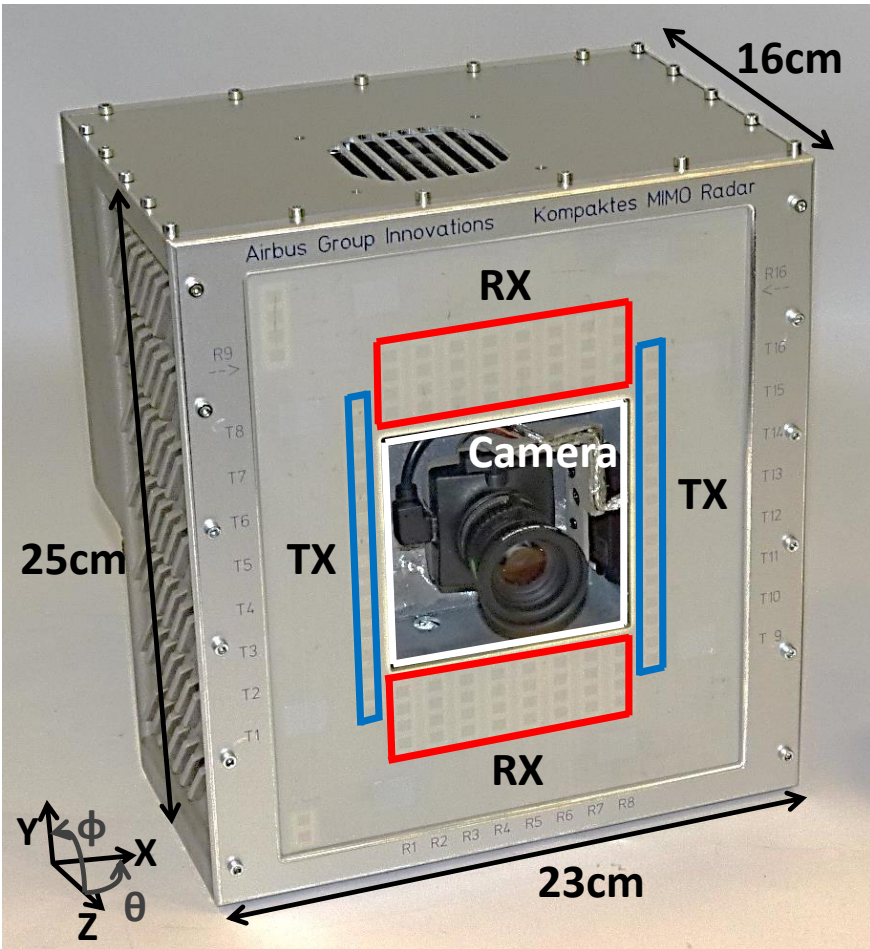

Fig. 4. Picture of the 3D printed housing with antenna board and camera mounted in the middle. The markers show where the TX and RX antennas, and the camera are placed.

at the right time or to lead the signal to a terminated port (50 $\Omega$ resistor) in order to accomplish a orthogonality of the received data streams under a TDM paradigm. A more detailed description of the antenna board can be found in [15].

The receiver board consists of 16 direct-conversion receivers. The RX signals are amplified by Low Noise Amplifiers (LNAs) and then fed to the mixers. A chirp distribution network splits up the chirp signal and distributes it coherently to all 16 stages. Each stage has its own amplifier in order to drive the LO input port of the mixer. The RX signal is then mixed with the originally sent chirp and low-pass filtered. From the output of the receiver, the Intermediate Frequency (IF) signals go directly to the digital board.

The main elements of the digital board are the ZYNQ which is a FPGA and an ARM processor in one chip, two 8channel 14-bit ADCs, which run at $100 \mathrm{MHz}$, and an Ethernet interface.

For the radar system to be compact and multifunctional, a lightweight, robust and adaptable housing is needed. To fulfill these requirements, a special housing was designed and 3D printed in aluminum. Additionally to the protection of the components, the housing is used as a heat sink. All boards are thermally linked to the housing and two cooling channels which are connected to a fan enhance the heat dissipation of the system. Fig. 4 shows a photograph of the overall system with 3D printed housing. The boxes show the TX and RX elements, the camera and the two axis gimbal, which is mounted in the middle of the rectangular array. The size of the

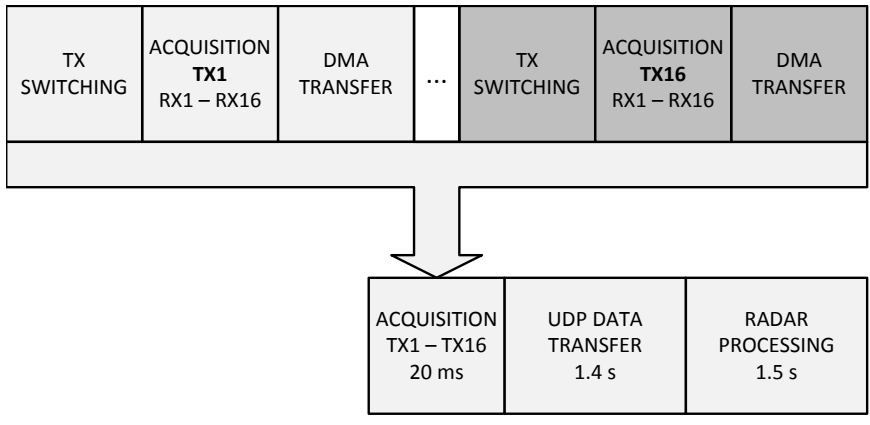

Fig. 5. Exemplary timing of a MIMO cycle

housing is $23 \mathrm{~cm} \times 25 \mathrm{~cm} \times 16 \mathrm{~cm}$. On the top of the housing, the opening of the cooling system can be seen.

\section{WAVEFORM AND TIMING}

This section describes the waveform and the timing of the MIMO radar. The system uses linear chirps from 16 -17 GHz. The up-ramp $\left(t_{u p}=100 \mu \mathrm{s}\right)$ is followed by a down-ramp $(20 \mu \mathrm{s})$, both form a triangular chirp. The down-ramp is not considered in the radar processing yet. Considering the upramp with a bandwidth $\mathrm{B}=1 \mathrm{GHz}$ and $c_{0}$ as the speed of light, a range resolution of

$$
\Delta_{R}=\frac{c_{0}}{2 B}=0.15 \mathrm{~m}
$$

is obtained. The maximum range of the system can be calculated using the sampling frequency of the ADCs $\left(f_{s}=100 \mathrm{MHz}\right)$ which determines the maximum difference frequency due to the sampling theorem.

$$
R_{\text {max }}=\frac{c_{0} \frac{f_{s}}{2}}{2 \frac{B}{t_{u p}}}=750 \mathrm{~m}
$$

Here, a MIMO cycle is composed of 16 up-ramps, where each ramp is sent via a different TX antenna. In order to avoid interferences between the TX signals and then achieve orthogonality, TDM is used. With TDM it is possible to assign the TX antenna to the RX signal according to the timing scheme.

The timing of a MIMO cycle is shown in Fig. 5. At the beginning, the system waits for a start command from the HMI. After that, the first TX antenna is activated and sends the FMCW chirp. The RX signals are captured by the ADCs and stored in the FPGA. After the that, the data is transfered from the FPGA to the DDR memory of the system via DMA. This procedure is repeated for all $16 \mathrm{TX}$ antennas and lasts, altogether, approximately $20 \mathrm{~ms}$. Currently, the radar beamforming algorithms are carried out in a MATLAB environment. For this reason, the data of the complete MIMO cycle is transferred via Ethernet to a workstation. After the transfer, the radar processing is launched. With the current configuration the system has an update rate of about $3 \mathrm{~s}$. This is mainly determined by the User Datagram Protocol (UDP) data transfer $(1.4 \mathrm{~s})$ and the MATLAB radar processing, which 


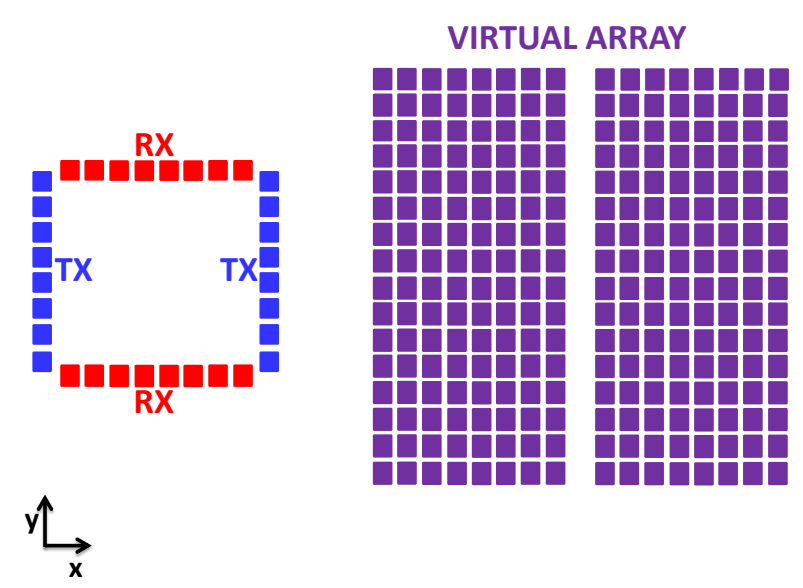

Fig. 6. Schematic representation of the MIMO antenna configuration with the physical array (left) showing the $16 \mathrm{TX}$ antennas in blue, the $16 \mathrm{RX}$ antennas in red and the resulting virtual array (right). A unit in the graph is equivalent to $\frac{d_{x}}{2}$ and $\frac{d_{y}}{2}$ respectively.

represent the current bottleneck of the system. These processes can be accelerated by implementing the radar processing directly in the FPGA.

\section{MIMO VIRTUAL ARRAY AND DIGITAL BEAMFORMING}

In the following section, the advantages of a MIMO radar are shown and the theoretical angular resolution is calculated. The MIMO radar has $16 \mathrm{TX}\left(N_{T X}\right)$ and $16 \mathrm{RX}\left(N_{R X}\right)$ antennas, leading to a total number of $N_{T X}+N_{R X}=32$ antennas and $N_{v i r}=N_{T X} N_{R X}=256$ virtual elements. The physical antenna placement and the resulting virtual array are shown in Fig. 6. It can be seen that the antenna elements are placed along a rectangle with 8 antennas per edge. The virtual array (in purple) can be calculated as the discrete convolution of all RX (in red) and TX (in blue) antenna element positions. To achieve the same angular resolution with a conventional mechanically or electronically scanning radar, $256 \mathrm{RX}$ antennas (16 x 16) and one TX antenna are needed. With a rectangular MIMO array configuration, the size of the antenna array can be reduced by a factor of two in both, $x$ - and $\mathrm{y}$-dimension, compared to a conventional phased array radar. Due to physical placement constraints, there is one additional column in y-direction, which is unoccupied. For the empty column which appears in the center of Fig. 6, an interpolation of the data is performed, according to what has previously been described in [16]. This results in a virtual array, which has 16 elements in x-direction $\left(N_{v i r, x}\right)$ and 17 elements in $\mathrm{y}$-direction $\left(N_{v i r, y}\right)$. The angular resolution defined as the 3dB-beamwidth of the main lobe for this particular MIMO array can be calculated as follows:

$$
\Delta \theta_{3 d B_{x}} \approx 50^{\circ} \frac{\lambda_{0}}{N_{v i r, x} d_{x}}=50^{\circ} \frac{\lambda_{0}}{\left(2 N_{p o p}+1\right) d_{x}} \approx 4.5^{\circ}
$$

$$
\Delta \phi_{3 d B_{y}} \approx 50^{\circ} \frac{\lambda_{0}}{N_{v i r, y} d_{y}}=50^{\circ} \frac{\lambda_{0}}{2 N_{\text {pop }} d_{y}} \approx 3.5^{\circ}
$$

with $\lambda_{0} \approx 18 \mathrm{~mm}$ being the wavelength, $d_{x}=12 \mathrm{~mm}$ and $d_{y}=16 \mathrm{~mm}$ the spacings between elements along the corresponding axis and $N_{\text {pop }}=8$ the number of antenna elements which are used along each edge of the rectangle.

The data structure for the MIMO processing is a real-valued three-dimensional data matrix $D \in \mathbb{R}^{N_{v i r, x} \times N_{v i r, y} \times M}$, where $N_{v i r, x} \times N_{v i r, y}$ is the dimension of the resulting virtual array which represents all TX-RX-combinations (including element interpolation). The elements in the first two dimensions have to be ordered in the same way as they result from the convolution. The third dimension $M$ results from the chirp length $(100 \mathrm{~ms})$ and the sampling frequency $(100 \mathrm{MHz})$ of the ADCs which results in $M=10000$ samples for each TX-RX-combination.

The 3D reconstruction of the radar scenario is done with a three-dimensional FFT on the data matrix described before. The FFT processing is very fast on the one hand, but requires a plane wavefront on the other hand. The first step is to perform an FFT along the third dimension $(M)$ of $D$. The result $\left(D_{1}\right)$ is a complex-valued range-compressed matrix for every element of the virtual array. Now the Direction of Arrival (DOA) of the targets can be estimated with two additional FFTs. For the azimuth angle extraction, an FFT across the second dimension $\left(N_{v i r, x}\right)$ of the previously calculated matrix $\left(D_{1}\right)$ is performed. The resulting matrix is $D_{2}$. Similarly, the elevation angles can be determined via an FFT across the third dimension $\left(N_{v i r, y}\right)$ of matrix $\mathrm{D}_{2}$.

The next paragraph explains the link between the result of the FFT and the DOA estimation. Here, just the calculations for the azimuth angles are presented, but the same principle applies for the elevation direction. For complex input samples $\left(D_{1}\right)$, the FFT results in a complex spectrum (two-sided). These complex points are sampled at the virtual element positions which leads to a sampling frequency in space of

$$
f_{s, \text { space }}=\frac{1}{d_{x}} \approx 83.3 \mathrm{~m}^{-1}
$$

and a frequency spacing for the FFT of

$$
d f_{\text {space }}=\frac{f_{\text {s,space }}}{N_{\text {vir }, x}} \approx 4.9 \mathrm{~m}^{-1}
$$

The FFT will give $N_{v i r, x}$ bins equally distributed from $f=0$ to $f=f_{s, \text { space }}$. Let $\alpha$ be the angle between the incident wavefront and the antennas, spanning between $-90^{\circ}$ to $90^{\circ}$. This means that the first sample is the component which belongs to a straight incident wave $\alpha=0$. The samples above $\frac{f_{s, s p a c e}}{2}$ represent negative angles and have to be shifted according to Tab. I. The reordering of the FFT leads to a representation with $\alpha=0^{\circ}$ in the center.

For each frequency sample $n$ of the FFT, the corresponding angle can be calculated as:

$$
\alpha=\arcsin \left(n \cdot d f_{\text {space }} \cdot \lambda_{0}\right)
$$


TABLE I

FFT ORIGINAL AND REORDERED, INDEX AND CORRESPONDING ANGLE.

\begin{tabular}{|l|c|c|c|c|c|c|c|c|c|}
\hline Original & 1 & 2 & $\ldots$ & 8 & 9 & 10 & $\ldots$ & 16 & 17 \\
\hline \hline Reordered & 10 & 11 & $\ldots$ & 17 & 1 & 2 & $\ldots$ & 8 & 9 \\
\hline Index $\mathrm{n}$ & -8 & -7 & $\ldots$ & -1 & 0 & 1 & $\ldots$ & 7 & 8 \\
\hline Angle in deg & -45 & -39 & $\ldots$ & -5 & 0 & 5 & $\ldots$ & 39 & 45 \\
\hline
\end{tabular}

with

$$
\left\{n \in \mathbb{N} \mid-\frac{N_{v i r, x}-1}{2} \leq n \leq \frac{N_{v i r, x}-1}{2}\right\}
$$

This leads to azimuth angles from $-45^{\circ}$ to $45^{\circ}$ (see Tab. I) for the system.

\section{SYSTEM PERFORMANCE VERIFICATION AND MEASUREMENT RESULTS}

This section is divided into two parts, the verification of the system performance and the presentation of measurement results for an application. The angular variables are $\theta$ for azimuth and $\phi$ for elevation. The coordinate system is displayed in Fig. 4.

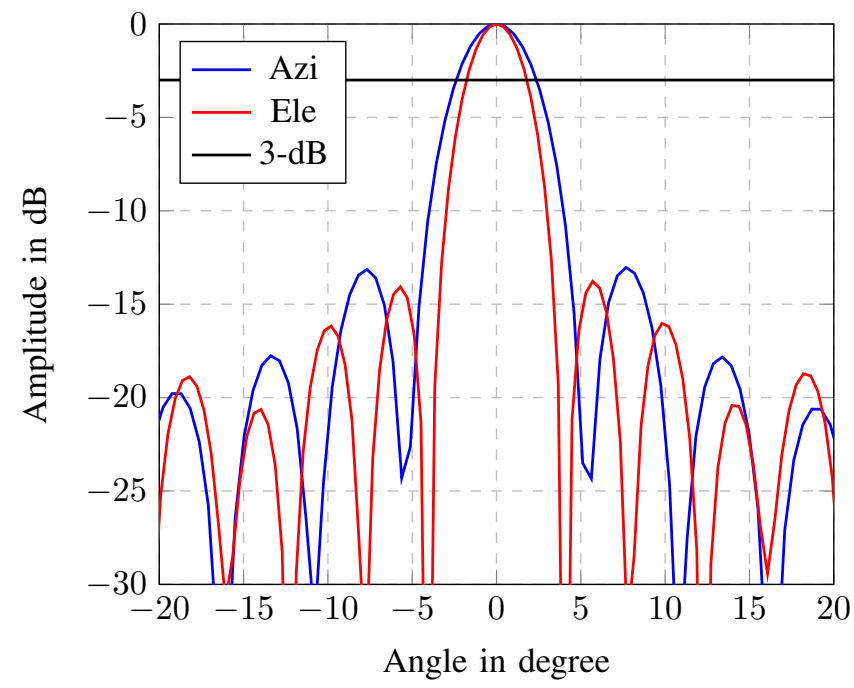

Fig. 7. Azimuth and elevation profiles for a central target.

\section{A. Angular resolution}

In order to measure the 3-dB-beamwidth of the system, a corner cube with a cross-section of $\sigma_{0}=150 \mathrm{~m}^{2}$ is placed in an anechoic chamber, at a range $R=23.1 \mathrm{~m}$, with azimuth and elevation angles of $\phi=0^{\circ}$ and $\theta=0^{\circ}$, respectively. With the FFT processing and FMCW ramp settings presented in former sections, a three-dimensional radar image, which contains the information of range, azimuth and elevation, is calculated. In order to measure the 3-dB-beamwidth of the system, a corner cube with a cross-section of $\sigma_{0}=150 \mathrm{~m}^{2}$ is placed in an anechoic chamber, at a range $R=23.1 \mathrm{~m}$, with azimuth and elevation angles of $\phi=0^{\circ}$ and $\theta=0^{\circ}$, respectively. With the FFT processing and FMCW ramp settings presented in former sections, a three-dimensional radar image, which contains the information of range, azimuth and elevation, is calculated.

Fig. 7 shows the normalized azimuth $\left(\phi=0^{\circ}\right)$ and elevation $\left(\theta=0^{\circ}\right)$ profiles taken at the range cell of the central target. Under the assumption that the corner cube is a point target and the distance is big enough to be in the far-field region, the theoretical values of the angular resolution of the MIMO virtual array (calculated in Section V) should match with the measured 3-dB-beamwidth, shown in Fig. 7. The measured azimuth resolution is $\Delta \theta_{3 d B_{x}}=4.7^{\circ}$ and the elevation resolution is $\Delta \phi_{3 d B_{y}}=3.6^{\circ}$ whereas the calculated is $\Delta \theta_{3 d B_{x}}=4.5^{\circ}$ and the elevation resolution is $\Delta \phi_{3 d B_{y}}=3.5^{\circ}$. As it can be seen, the measured and calculated angular resolutions match very well. One thing to consider is that in the estimation along the azimuth direction the missing element is included, while in the radar beam-forming process, this missing element is calculated as the average of its neighbors.

\section{B. Radar field of view}

The aim of this sub-chapter is to show the dependency between a target's received power, at a certain distance $R$, and its angle, in both azimuth and elevation directions.

A corner cube with a cross-section of $\sigma_{0}=150 \mathrm{~m}^{2}$ is located in an anechoic chamber. First, the radar is rotated until the imaginary line, which crosses the radar and the target, is set to be $\phi=-\phi_{\max }$ and $\theta=0$. Then a radar measurement with the previously presented parameters is started. This process is repeated following an angular sweep from $\phi=-\phi_{\max }, \theta=0^{\circ}$ to $\phi=\phi_{\max }, \theta=0^{\circ}$ (always with constant $R=23.1 \mathrm{~m}$ ). Since the $\mathrm{RX}$ (array of 4 patches along y) and TX (array of 2 patches along y) antennas are not isotropic, a relatively pronounced dependency of the received power with the angle is expected.

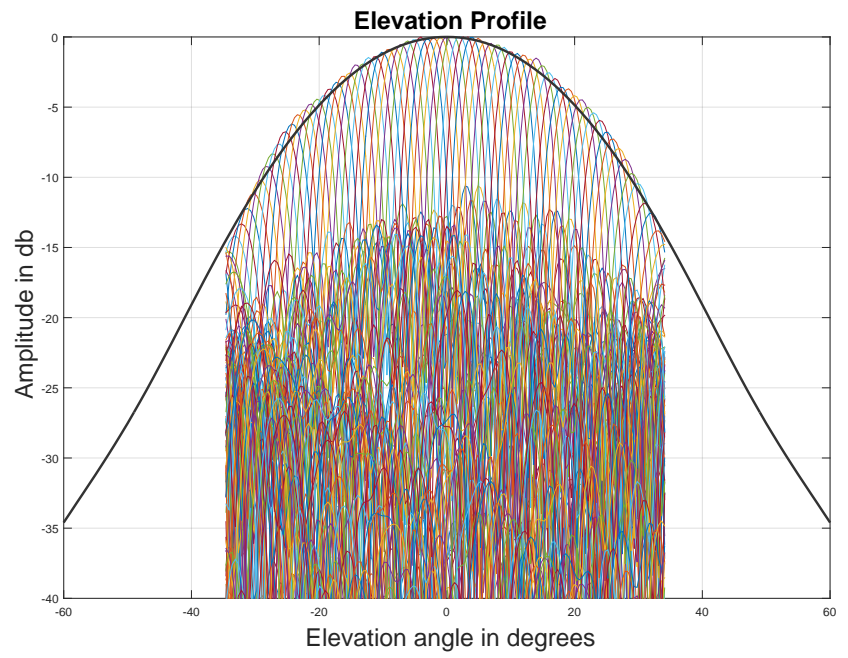

Fig. 8. Normalized dependence of the received power in the elevation direction with the angle due to the antenna element pattern.

Fig. 8 illustrates the measured curves for different angles $\phi=\phi_{i}, \theta=0^{\circ}$. The envelope of these curves is the 
multiplication of the simulation of the RX antenna pattern and the TX antenna pattern. The amplitude decays approximately within $\pm 10 \mathrm{~dB}$ in $30^{\circ}$. The simulation matches very well with the performed measurement.

A second analog procedure has been conducted. This time the angular sweep is from $\phi=0^{\circ}, \theta=-\theta_{0}$ to $\phi=0^{\circ}$, $\theta=\theta_{0}$ (the distance to the target was still $R=23.1 \mathrm{~m}$ ). Since the RX (single patch along $\mathrm{x}$ ) and TX (single patch along $\mathrm{x}$ ) antennas are not isotropic, a corresponding angular dependence of the power is expected. In this axis the antennas are single patches, therefore, the characteristic dependence of the received power with the angle is expected to be rather wide.

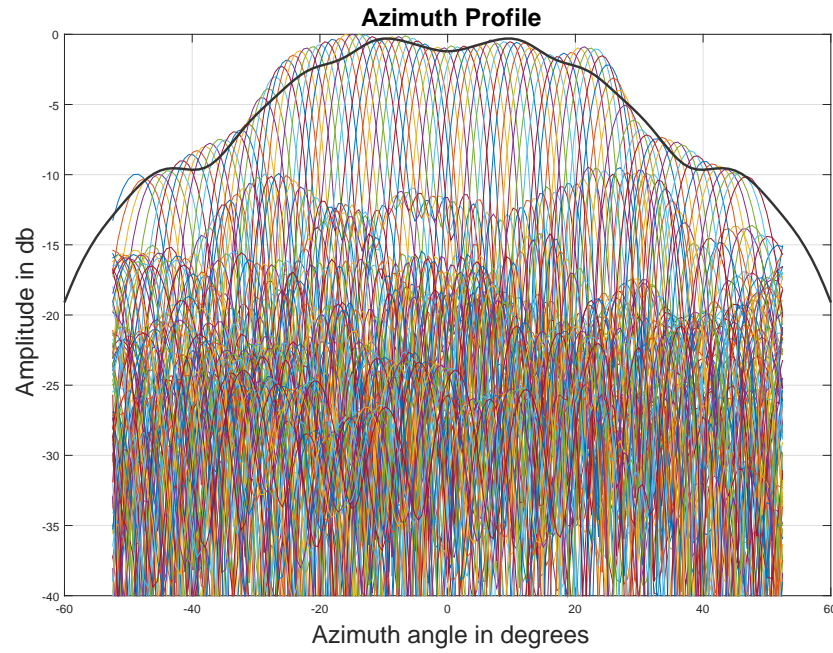

Fig. 9. Normalized dependence of the received power in the azimuth direction with the angle due to the antenna element pattern.

Fig. 9 illustrates the measured curves for different angles $\left(\phi=\phi_{i}, \theta=0^{\circ}\right)$. In order to accomplish the TDM paradigm, each TX radiating element is connected to a switch, in such a manner that only the TX antenna which sends the FMCW ramp is matched to $50 \Omega$, while the rest of the TX antennas are connected to a short-circuit. Consequently, a ripple appears in the elevation dimension. The envelope of these curves is the multiplication of the simulation of RX antenna pattern and the TX antenna pattern (considering the whole antenna array with the corresponding open circuits in the proper TX elements). The amplitude decays approximately $10 \mathrm{~dB}$ within $\pm 50^{\circ}$. The simulation matches rather well the performed measurement. As a summary, the FOVs of the radar are $F O V_{\theta}=100^{\circ}$ and $F O V_{\theta}=60^{\circ}$, respectively.

\section{Zone surveillance applications}

The next step is to verify the performance of the MIMO radar in a real scenario and to show the advantages of a multifunctional system. In this test, the MIMO radar is combined with a camera which is placed in the middle of the antenna array. Fig. 10 shows the scene of the measurement as

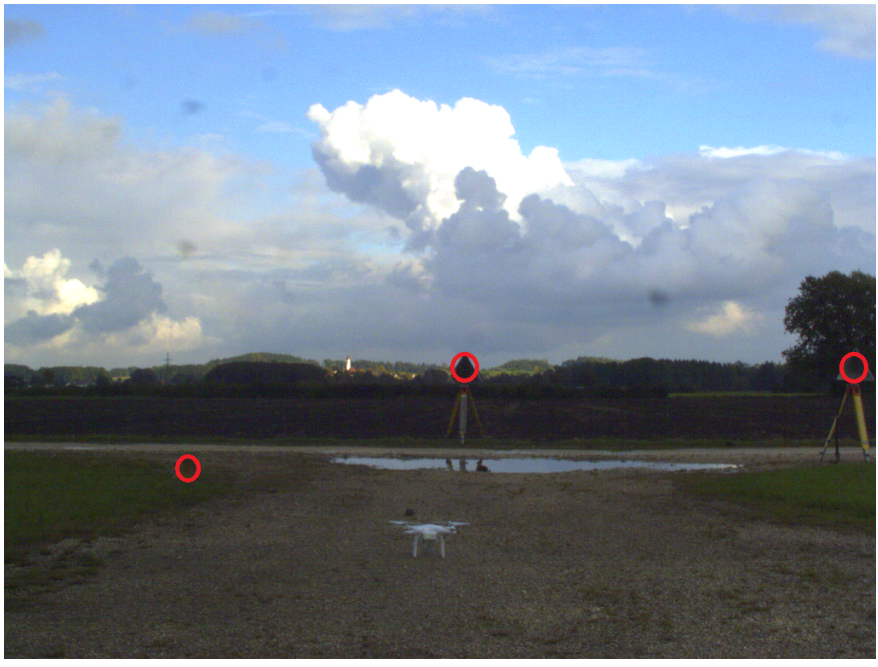

Fig. 10. Image of the scene with three corner cubes marked with red circles.

seen by the camera. A commercial camera, with $n_{p i x, h}=2048$ pixels horizontally and $n_{p i x, v}=1536$ pixels vertically is used. The pixel size is $3.2^{\circ}$. With an objective with a focal length of $f=12 \mathrm{~mm}$, a field of view of

$$
F O V_{h}=\frac{n \cdot p}{f} \cdot \frac{360^{\circ}}{2 \pi}=31.3^{\circ}
$$

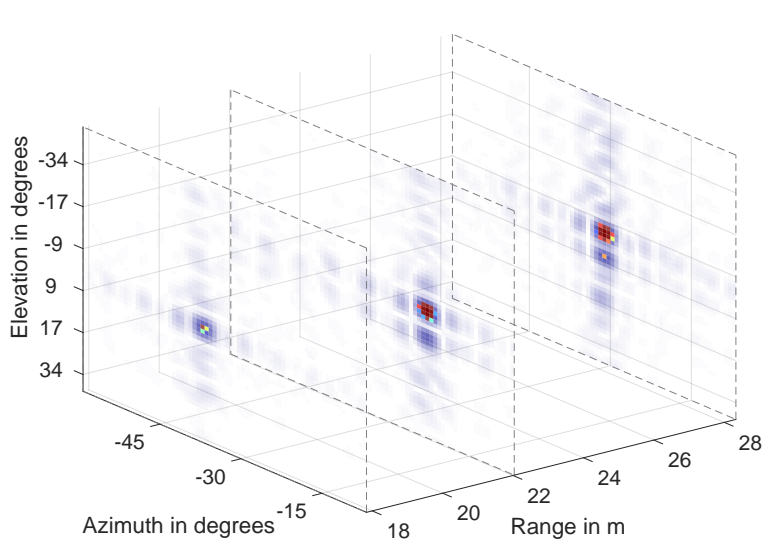

Fig. 11. 3D reconstruction of the scene with radar data. The three targets are spaced in range and have different positions in azimuth and elevation.

horizontally and $F O V_{v}=23.5^{\circ}$ vertically is obtained. The field of view of the radar have been calculated in the previous sub-section to be $F O V_{\phi}=60^{\circ}$ and $F O V_{\theta}=100^{\circ}$. Since the FOV of the radar is greater than the FOV of the camera, the gimbals can be used to point the camera to the right direction. A field test measurement has been performed, in order to simulate a detectable change in the surveillance area. For this purpose, three corner cubes with cross-sections $\sigma_{1} \approx 49 \mathrm{~m}^{2}$, $\sigma_{2} \approx 422 \mathrm{~m}^{2}$ and $\sigma_{3} \approx 169 \mathrm{~m}^{2}$ are placed at ranges of $18 \mathrm{~m}, 28 \mathrm{~m}$ and $22 \mathrm{~m}$, respectively. Determining the accurate 
position of the objects in a medium range is a computationally intensive task for pure image processing algorithms.

The results of the 3D-FFT beamforming radar processing proposed in this paper is shown in Fig. 11. As it can be seen, a correct estimation of the range, azimuth and elevation, for all 3 targets used in the measurement is achieved.

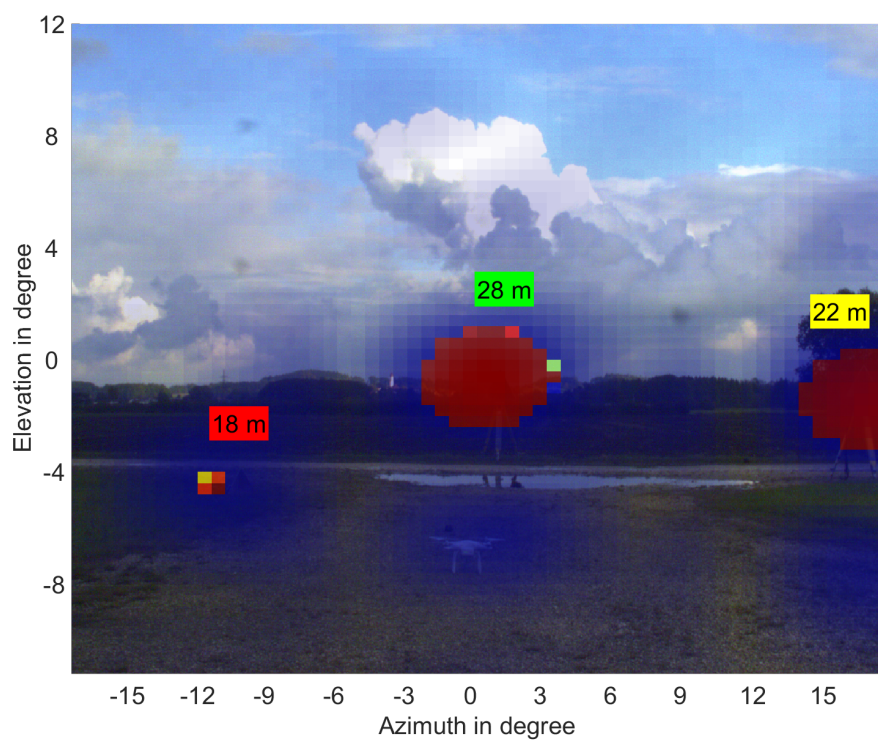

Fig. 12. Camera and radar image overlaid. The color and the level of opaqueness is proportional to the amplitude of the reflection. The targets' ranges are displayed in boxes with different colors. The field of view is limited by the camera.

An additional and more intuitively understandable image, fusion of the camera image and the radar data is presented in Fig. 12. Here, the camera image is superimposed with the radar data. In order to still see the scene, the amplitude of the reflection is used to define the transparency level and the color of the radar cell. With this method, strong reflections occur as opaque areas in the image. In this case, the evaluation is done for the three most significant targets in amplitude. According to the distance, a red, a yellow and a green tag are placed close to the targets, where the red tag shows the closest, and usually most important, target. This way of displaying the information is very intuitive since a strong target within the monitored scene is highlighted in the camera image. Hence, a higher degree of automation is achieved. Another aspect to remark is that the system is compact and portable and thus can be easily integrated into existing infrastructures.

Another application is the detection and classification based on image processing. In this case the radar can be combined with a zoom camera pointed to the objects previously detected by the radar. Therefore, a different camera objective is needed, e.g. with a focal length of $f=75 \mathrm{~mm}$, which leads to a field of view of $F O V_{h}=5.0^{\circ}$. This is comparable to the azimuth resolution of the radar, which makes it possible to observe each azimuth sector separately. To point the camera, the two axis gimbal can be used in combination with the already implemented tracking algorithm. The servos of the camera gimbal are linked to the radar processing and receive the azimuth and elevation angles of the most significant target. This could be the one with the highest amplitude or the one which is closest to the radar. The gimbal then moves to the target's direction and offers a much more confined area to be processed by the image processing. The zoom increases the resolution of the target. Object detectors in image processing need a certain minimum number of pixels, on which they can operate. When using a wide-angle objective, the resolution of a target in medium distance would be too low. With a zoom objective the resolution is increased and the probability of detection is higher and a better classification can be performed.

\section{CONCLUSION}

A compact, portable and multifunctional system, which combines an FMCW MIMO radar and a camera, has been introduced. The device has demonstrated to be suitable for the presented application (change detection in wide zones such as nuclear plants). Detailed hardware and software architecture descriptions have been presented together with a description of the DOA estimation and the MIMO cycle timing. The performance of the multifunctional system has been verified by measurements in a multi-target environment showing synergies among the two functionalities. The system is able to perform a $3 \mathrm{D}$ reconstruction of the surroundings via the MIMO radar, extracting range, azimuth and elevation of the targets, and combine this information with a camera image. This technological approach has a high potential, especially for these applications in which the space limitation is critical as for instance, landing aid for helicopters in brown-out/ white-out situations or for object classification in flying platforms.

\section{ACKNOWLEDGMENT}

\section{REFERENCES}

[1] Fasano, G., Accardo, D., Moccia, A., Carbone, C., Ciniglio, U., Corraro, F. and Luongo, S. (2008). Multi-sensor-based fully autonomous noncooperative collision avoidance system for unmanned air vehicles. Journal of aerospace computing, information, and communication, 5(10), 338360

[2] J. S. Dittrich and E. N. Johnson, "Multi-sensor navigation system for an autonomous helicopter," Proceedings. The 21st Digital Avionics Systems Conference, 2002, pp. 8C1-1-8C1-19 vol.2.

[3] D. L. Hall and J. Llinas, "An introduction to multisensor data fusion," in Proceedings of the IEEE, vol. 85, no. 1, pp. 6-23, Jan 1997.

[4] M. Skolnik, "Radar Handbook, Third Edition", ser. Electronics electrical engineering. McGraw-Hill Education, 2008.

[5] Y. Huang, P. V. Brennan, D. Patrick, I. Weller, P. Roberts, and K. Hughes, "FMCW based MIMO imaging radar for maritime navigation", in Progress In Electromagnetics Research, Vol. 115, 327-342, 2011.

[6] Zhengping Ji and D. Prokhorov, "Radar-vision fusion for object classification," 2008 World Automation Congress, Hawaii, HI, 2008, pp. 1-6.

[7] S. Kemkemian, M. Nouvel-Fiani, P. Cornic and P. Garrec, "A MIMO radar for Sense and Avoid function: A fully static solution for UAV,", in 11-th Int. Radar Symp., Vilnius, Lithuania, 2010, pp. 1-4.

[8] G. M., Brooker, "Understanding millimetre wave FMCW radars.", in 1st international Conference on Sensing Technology, 2005.

[9] A. J. Kirschner, J. Guetlein, S. Bertl and J. Detlefsen, "A millimetrewave MIMO radar system for threat detection in patrol or checkpoint scenarios," IET International Conference on Radar Systems (Radar 2012), Glasgow, UK, 2012, pp. 1-6. 
[10] R. Streubel and B. Yang, "Fusion of stereo camera and MIMOFMCW radar for pedestrian tracking in indoor environments," 2016 19th International Conference on Information Fusion (FUSION), Heidelberg, 2016, pp. 565-572.

[11] G. C. Tavik et al., "The advanced multifunction RF concept," in IEEE Transactions on Microwave Theory and Techniques, vol. 53, no. 3, pp. 1009-1020, March 2005.

[12] E. Brookner. "Phased-array radars.", Scientific American 252.2, pp. 94$102,1985$.

[13] J. Li, P. Stoica, "MIMO Radar Signal Processing", John Wiley \& Sons, Inc., 2008.

[14] F. Croq and A. Papiernik, "Large bandwidth aperture coupled microstrip antenna", in Electronics Letters, vol. 26, pp. 1293-1294, August 1990.

[15] E. Miralles, D. Rieth, B. Schoenlinner, U. Prechtel, A. Meusling, A. Belenguer, H. Esteban and V. Ziegler, "Low Cost Multipurpose RF System Approach: Integration of a MIMO Radar and a Communications Frontend on a Single PCB", in IEEE Microwave Conference (EuMC), 2016 European, London, UK, Oct 2016.

[16] Spreng, T., Shuai Yuan, Valenta, V., Schumacher, H., Siart, U., Ziegler, V., "Wideband $120 \mathrm{GHz}$ to $140 \mathrm{GHz}$ MIMO radar: System design and imaging results", in Microwave Conference (EuMC), 2015 European, pp. 430-433, 7-10 Sept. 2015 May 2012. 